\title{
SpS1-Gas in protoplanetary disks
}

\author{
Miwa Goto ${ }^{1}$ \\ ${ }^{1}$ Max-Planck-Institut für Astronomie, Königstuhl 17, Heidelberg, D-69117, Germany
}

\section{Introduction}

High resolution infrared spectroscopy is the key technique to look at the inner regions of protoplanetary disks. As molecular hydrogen is an inefficient emitter, $\mathrm{CO}$ gas is the single most important molecular probe of the disk. The energy gaps of the vibrationally excited levels $(\Delta E>3000 \mathrm{~K})$ and the critical density required to keep the molecules in the excited state $\left(n_{c} \sim 10^{10} \mathrm{~cm}^{-3}\right)$ match well to the physical condition of the inner regions of protoplanetary disks. In order to resolve the vibrational lines of different rotational states, a spectral resolving power of $\lambda / \Delta \lambda>10000$ is necessary; or even higher ( $>30000-100000)$, if we would like to fully resolve the gas kinematics. Scoville et al. (1980) provided the fundamentals of the excitation mechanisms, which is essential for the interpretation of the vibrational transitions of CO, and pioneered the study of the circumstellar environment with infrared CO lines in the observation of BN (Scoville et al. (1983)). The bandhead emission of $\mathrm{CO}$ at $2.3 \mu \mathrm{m}$ from young stars was unambiguously attributed to the circumstellar disks by Carr (1989) and Najita et al. (1996), because the gas kinematics matches well to what is expected from Keplerian rotation. Since then, the gas kinematics have been extensively used to shed light on peculiar disk structures, such as the inner truncation (Brittain et al. 2003), the outer truncation (Najita et al. 2008), and the gap (van der Plas et al. 2008; though this is an oxygen forbidden line).

\section{High angular resolution}

The most notable breakthrough in $\mathrm{CO}$ observation in the past decade was the advent of $8-\mathrm{m}$ class telescopes equipped with adaptive optics systems. The high angular resolution attained by these new devices allows us to spatially resolve the line emission of a few nearby disks, without relying on the line kinematics. This can be accomplished by either the spectroastrometric technique (Pontoppidan et al. 2008; van der Plas et al. 2009) or in a fully resolved manner as in the case of CO line emission from the Herbig Ae star HD 141569A (Goto et al. 2006). HD 141569A is a transition object in the class of Herbig Ae/Be stars with little infrared excess at wavelengths shorter than $10 \mu \mathrm{m}$. The line images of its disk show that the inner disk is apparently truncated at a radius of $11 \mathrm{AU}$, which is about half of the nominal gravitational radius of the star. The size of the inner cavity, which was too large to be accounted for by dust sublimation or magnetospheric truncation, strongly indicates that the inner disk is removed by photoevaporation (Clarke et al. 2001).

\section{Spectroastrometry}

Spectroastrometry is an alternative way to squeeze out spatial information from protoplanetary disks by measuring the centroid of an emission line for various wavelengths. In this technique, the size of the point-spread function is not the hard limit of the accuracy of the measurement anymore, but the signal-to-noise ratio of the emission lines. With an integral field spectrograph, spectroastrometry can be done even in two dimensions. An example is the spectroastrometry of $\mathrm{Br} \gamma$ of TW Hya performed by SINFONI at the VLT (Goto et al. 2009, submitted). The centroid of the emission line is measured to an accuracy of a hundredth of a pixel, making it possible to resolve the gas kinematics on the physical scale of the radius of the star. 


\section{Temporal Development}

High resolution $\mathrm{CO}$ observations are also used to observe the transient phenomena. Pre-main sequence stars are all more or less photometrically variable. The most outstanding variables among them are the outbursts of FU Ori or EX Lup type stars, where the young stars brighten up by several magnitudes in visible light, once in a decade to a century. EX Lup started its biggest outburst on record in early 2008. The gas kinematics during the outburst were monitored at CO $4.7 \mu \mathrm{m}$ for five months for a total of six epochs. The observations revealed that the inner disk is cut off at $0.2-0.3 \mathrm{AU}$ inward and drained out, while the outer disk does not change. Toward the end of the massive accretion phase, the line profile varied rapidly with a flipping of the asymmetry on timescales of a few days to a week, suggesting that a hot spot emerged on the orbiting gas disk (Goto et al. 2009 in prep.).

\section{Future Direction}

METIS is one of the infrared instruments currently being proposed for E-ELT. It offers imaging and spectroscopic capabilities at 3-14 $\mu \mathrm{m}$ and is fully compatible with the adaptive optics system. The most notable feature of the instrument is the integral field spectroscopy at $3-5 \mu \mathrm{m}$. With an improvement of the angular resolution by a factor of five, this instrument will be the cornerstone to the next breakthrough in the understanding of the physical and chemical evolution of the gas in protoplanetary disks.

\section{References}

Brittain, S. D. et al. 2003, ApJ, 588, 535

Carr, J. S. 1989 ApJ, 345, 522

Clarke, C. J., Gendrin, A., \& Sotomayor, M. 2001, MNRAS, 328, 485

Goto et al., 2006, ApJ, 652, 758

Najita, J., Carr, J. S., Glassgold, A. E., Shu, F. H., \& Tokunaga, A. T. 1996, ApJ, 462, 919

Najita, J. R., Crockett, N., \& Carr, J. S. 2008, ApJ, 687, 1168

Pontoppidan, K. M., et al., 2008, ApJ, 684, 1323

Scoville, N., Kleinmann, S. G., Hall, D. N. B., \& Ridgway, S. T. 1983, ApJ, 275, 201

Scoville, N. Z., Krotkov, R., \& Wang, D. 1980, ApJ, 240, 929

van der Plas, G. et al., 2008, A\& $A$, 485, 487

van der Plas, G. et al., 2009, A\&\&A, 500, 1137 